\title{
Linking Macro- and Micro-Structural Analysis with Luminescence Control in Oxynitride Phosphors for Light- Emitting Diodes
}

Mu-Huai Fang, ${ }^{\dagger}$ Jia-Cheng Lin, ${ }^{\dagger}$ Wen-Tse Huang, ${ }^{\dagger}$ Natalia Majewska, ${ }^{\S}$ Justyna Barzowska, ${ }^{\S}$ Sebastian Mahlik, ${ }^{\S}$ Wei Kong Pang, ${ }^{\Phi}$ Jyh-Fu Lee, ${ }^{\psi}$ Hwo-Shuenn Sheu, ${ }^{\Psi}$ and Ru-Shi Liu ${ }^{\dagger}, *$

Department of Chemistry, National Taiwan University, Taipei 106, Taiwan

$\S$ Institute of Experimental Physics, Faculty of Mathematics, Physics and Informatics, University of Gdańsk, Wita Stwosza 57, 80-308 Gdańsk, Poland

${ }^{\Phi}$ Institute for Superconducting \& Electronic Materials, University of Wollongong, NSW 2522, Australia

${ }^{\Psi}$ National Synchrotron Radiation Research Center, Hsinchu 300, Taiwan 


\section{EXPERIMENTAL METHODS}

Reagents. Strontium oxide ( $\mathrm{SrO} ; 99.9 \%)$, aluminum oxide $\left(\mathrm{Al}_{2} \mathrm{O}_{3} ; 99 \%\right)$, and lithium carbonate $\left(\mathrm{Li}_{2} \mathrm{CO}_{3} ; 99.997 \%\right)$ were obtained from Aldrich. Lithium nitride $\left(\mathrm{Li}_{3} \mathrm{~N}\right.$; $99.5 \%)$ was purchased from Materion. Europium oxide $\left(\mathrm{Eu}_{2} \mathrm{O}_{3} ; 99.995 \%\right)$ was acquired from Alfa Aesar.

Synthesis of $\mathrm{Sr}_{0.98} \mathrm{Li}_{\mathbf{2 . 5 + 2}} \mathrm{Al}_{\mathbf{1 . 5 - 2}} \mathrm{O}_{\mathbf{3}+2 \mathrm{z}} \mathrm{N}_{\mathbf{1 - 2 z}}: \mathbf{0 . 0 2 E u}$. All experiments were carried out in an inert gas-filled glovebox $\left(\mathrm{O}_{2}<1 \mathrm{ppm}, \mathrm{H}_{2} \mathrm{O}<1 \mathrm{ppm}\right)$ due to the moisture-sensitive nature of the starting materials. The starting materials were mixed in an agate mortar and placed into nickel crucibles, which were then placed in a tube furnace. Forming gas $\left(10 \% \mathrm{H}_{2}+90 \% \mathrm{~N}_{2}\right)$ was used to reduce $\mathrm{Eu}^{3+}$ into $\mathrm{Eu}^{2+}$. After heating the sample to 900 ${ }^{\circ} \mathrm{C}$ at a rate of $5{ }^{\circ} \mathrm{C} / \mathrm{min}$ under $1 \mathrm{~atm}$ pressure for $4 \mathrm{~h}$, the samples were cooled to room temperature to obtain $\mathrm{Sr}_{0.98} \mathrm{Li}_{2.5+\mathrm{z}} \mathrm{Al}_{1.5-\mathrm{z}} \mathrm{O}_{3+2 \mathrm{z}} \mathrm{N}_{1-2 \mathrm{z}}: 0.02 \mathrm{Eu}$ phosphors.

Characterization. The phase and purity of the as-prepared powder samples were examined by X-ray diffraction (XRD) analysis by a D2 PHASER diffractometer (Bruker) with $\mathrm{Cu} \mathrm{K} \alpha$ radiation source and the wavelength $\lambda=1.5418 \AA$. Synchrotron powder X-ray diffraction patterns of $\mathrm{Sr}_{0.98} \mathrm{Li}_{2.5+\mathrm{z}} \mathrm{Al}_{1.5-\mathrm{z}} \mathrm{O}_{3+2 \mathrm{z}} \mathrm{N}_{1-2 \mathrm{z}}: 0.02 \mathrm{Eu}$ were acquired from the National Synchrotron Radiation Research Center (NSRRC, Taiwan) BL01C2 beamline with the wavelength $\lambda=0.82657 \AA$ at room temperature (RT) by using a Debye-Scherrer camera. High-resolution neutron powder diffraction (NPD) data were collected from the ECHIDNA at the Open Pool Australian Light water (OPAL) research reactor at the Australian Nuclear Science and Technology Organisation (ANSTO, Australia) with the wavelength $\lambda=1.62182(26) \AA$ calibrated by La ${ }^{11} \mathrm{~B}_{6}$ NIST Standard Reference Material 660b. The diffraction patterns from synchrotron XRD and NPD were used for Rietveld analysis using Total Pattern Analysis Solutions software (TOPAS 5.0). The ratio of $\mathrm{Eu}^{2+} / \mathrm{Eu}^{3+}$ was checked by $\mathrm{Eu} L_{3}$-edge of X-ray absorption near-edge structure (XANES) spectroscopy in fluorescence mode at the BL01C1 beamline in NSRRC, Taiwan. The Eu $L_{3}$-edge of extended X-ray absorption fine structure (EXAFS) experiments were conducted at the BL17C1 beamline in NSRRC, Taiwan. Solid-state nuclear magnetic resonance spectra were collected on a Bruker 600 $\mathrm{MHz}$ AVIII 600 WB NMR spectrometer. RT photoluminescence (PL) photoluminescence excitation (PLE) spectra were recorded with a FluoroMax-4P spectrofluorometer (Horiba) equipped with a $150 \mathrm{~W}$ xenon lamp as an excitation source and an R928 Hamamatsu photomultiplier as a detector, which enabled the recording of 
PL and PLE spectra within the spectral range of $250-850 \mathrm{~nm}$. The temperature- and pressure-dependent PL spectra were evaluated with an Andor SR-750-D1 spectrometer equipped with a CCD camera (DU420A-OE). A Kimmon Koha He-Cd laser (442 nm) was used as an excitation source. Decay profiles were obtained using time-resolved spectroscopy equipped with a PG 401/SH optical parametric generator pumped by a PL2251A pulsed YAG:Nd laser (EKSPLA). The detection part consisted of a $2501 \mathrm{~S}$ grating spectrometer (Bruker Optics) combined with a C4334-01 streak camera (Hamamatsu). Data were recorded in the form of streak images on a $640 \times 480$ pixel CCD array. Photon-counting algorithm-based software was used to transform the result into a 2D matrix of photon counts versus wavelength and time (streak image). Quantum yield was examined by Quantaurus-QY absolute PL quantum yield spectrometer (Hamamatsu) with an excitation source of $460 \mathrm{~nm}$. White-light LEDs were prepared by combining a blue LED chip with green $\left(\mathrm{Sr}_{2} \mathrm{SiO}_{4}: \mathrm{Eu}\right)$ and red $\left(\mathrm{Sr}_{0.98} \mathrm{Li}_{2.5+\mathrm{z}} \mathrm{Al}_{1.5-\mathrm{z}} \mathrm{O}_{3+2 \mathrm{z}} \mathrm{N}_{1-2 \mathrm{z}}: 0.02 \mathrm{Eu}\right.$ and $\left.\mathrm{SrLiAl}_{3} \mathrm{~N}_{4}: \mathrm{Eu}\right)$ phosphors. The optical properties of white-light LED were measured using a UV-VIS-near IR spectrophotocolorimeter (Everfine Photo-E-INFO Co., Ltd.). 

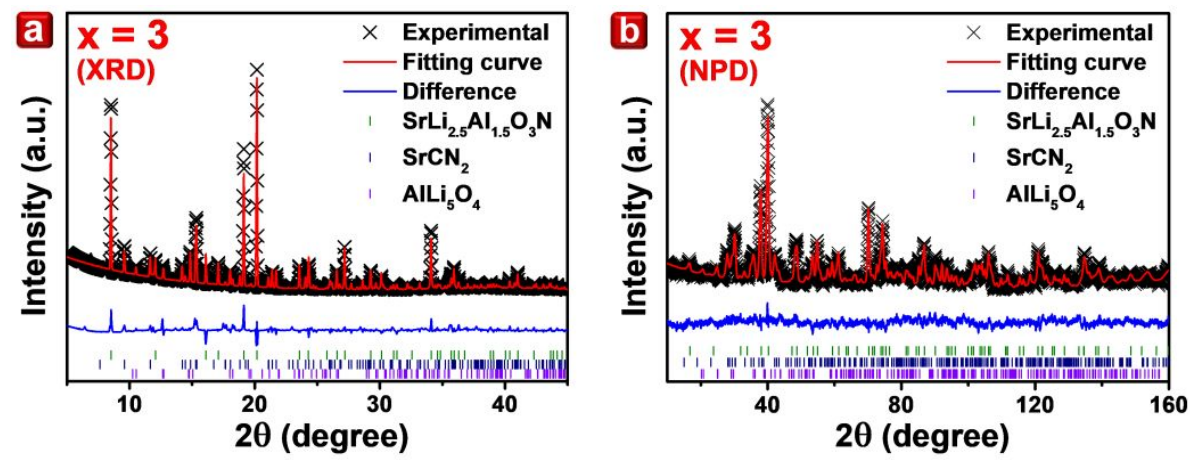

Figure S1. Joint refinement of (a) synchrotron X-ray diffraction and (b) neutron powder diffraction for $x=3$.
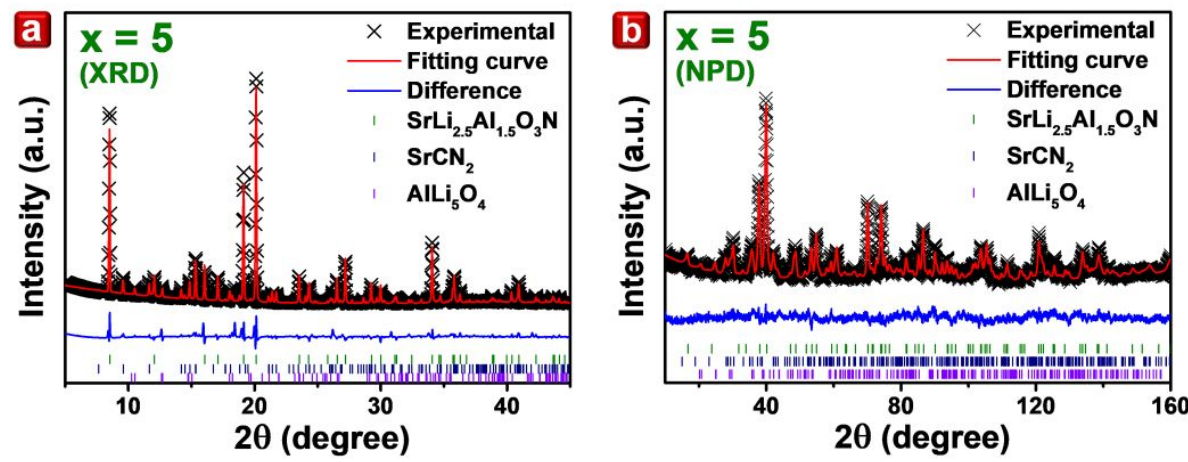

Figure S2. Joint refinement of (a) synchrotron X-ray diffraction and (b) neutron powder diffraction for $x=5$.
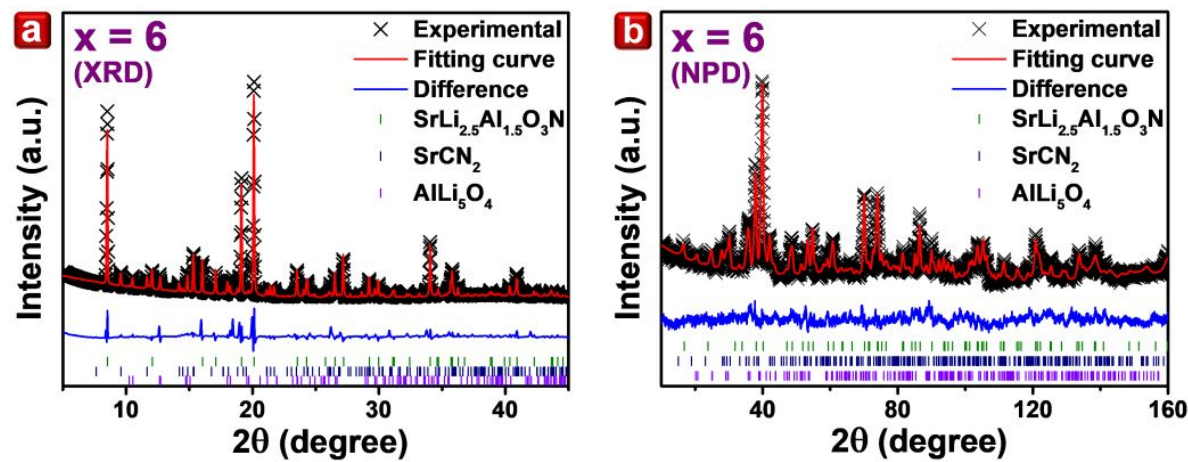

Figure S3. Joint refinement of (a) synchrotron X-ray diffraction and (b) neutron powder diffraction for $x=6$. 
Table S1. Results derived from the joint refinement for $x=3-6$.

\begin{tabular}{lcccc}
\hline \multicolumn{1}{c}{$\mathbf{x}=$} & $\mathbf{3}$ & $\mathbf{4}$ & $\mathbf{5}$ & $\mathbf{6}$ \\
\hline $\mathbf{a}=\mathbf{b}(\boldsymbol{A})$ & $7.861023 \pm 0.000130$ & $7.857921 \pm 0.000093$ & $7.857998 \pm 0.000122$ & $7.860216 \pm 0.000149$ \\
$\mathbf{c}(\boldsymbol{A})$ & $3.180855 \pm 0.000081$ & $3.187448 \pm 0.000062$ & $3.193795 \pm 0.000095$ & $3.196228 \pm 0.000104$ \\
$\mathbf{V}\left(\boldsymbol{A}^{3}\right)$ & $196.563 \pm 0.008$ & $196.815 \pm 0.006$ & $197.211 \pm 0.007$ & $197.473 \pm 0.010$ \\
Overall GOF & 2.90 & 2.52 & 3.28 & 3.72 \\
Overall $\mathbf{R}_{\text {wp }}$ (\%) XRD & 10.79 & 9.01 & 12.22 & 14.12 \\
Overall $\mathbf{R}_{\text {wp }}$ (\%) NPD & 4.40 & 4.31 & 4.41 & 4.30 \\
\hline
\end{tabular}

Table S2. Phase ratio determined by X-ray powder diffraction and neutron powder diffraction.

\begin{tabular}{|c|c|c|c|c|}
\hline \multicolumn{5}{|c|}{ Phase ratio in X-ray powder diffraction } \\
\hline Samples & $x=3$ & $x=4$ & $x=5$ & $x=6$ \\
\hline $\mathrm{SrLi}_{2.5+\mathrm{z}} \mathrm{Al}_{1.5-\mathrm{z}} \mathrm{O}_{3+2 \mathrm{z}} \mathrm{N}_{1-2 \mathrm{z}}$ & $69.32 \%$ & $74.79 \%$ & $76.49 \%$ & $71.70 \%$ \\
\hline $\mathrm{SrCN}_{2}$ & $18.38 \%$ & $14.01 \%$ & $12.17 \%$ & $11.53 \%$ \\
\hline $\mathrm{AlLi}_{5} \mathrm{O}_{4}$ & $12.30 \%$ & $11.20 \%$ & $11.34 \%$ & $16.76 \%$ \\
\hline \multicolumn{5}{|c|}{ Phase ratio in neutron powder diffraction } \\
\hline Samples & $x=3$ & $x=4$ & $x=5$ & $x=6$ \\
\hline $\mathrm{SrLi}_{2.5+z} \mathrm{Al}_{1.5-\mathrm{z}} \mathrm{O}_{3+2 \mathrm{z}} \mathrm{N}_{1-2 \mathrm{z}}$ & $68.76 \%$ & $74.12 \%$ & $74.87 \%$ & $69.87 \%$ \\
\hline $\mathrm{SrCN}_{2}$ & $17.41 \%$ & $13.28 \%$ & $11.43 \%$ & $10.78 \%$ \\
\hline $\mathrm{AlLi}_{5} \mathrm{O}_{4}$ & $13.84 \%$ & $12.60 \%$ & $13.70 \%$ & $19.35 \%$ \\
\hline
\end{tabular}

Table S3. Atomic positions, occupancy, and thermal parameters for $x=3$.

\begin{tabular}{cccccc}
\hline $\mathbf{x}=\mathbf{3}$ & $\boldsymbol{x}$ & $\boldsymbol{y}$ & $\boldsymbol{z}$ & Occ. & Beq $\left(\boldsymbol{A}^{2}\right)$ \\
\hline $\mathbf{S r}$ & 0.500000 & 0.500000 & 0.500000 & 1 & $3.10245 \pm 0.05299$ \\
$\mathbf{A l}$ & $0.19511 \pm 0.00088$ & $0.64081 \pm 0.00087$ & 0.000000 & 0.38650 & 1 \\
$\mathrm{Li}$ & $0.19511 \pm 0.00088$ & $0.64081 \pm 0.00087$ & 0.000000 & 0.61350 & 1 \\
$\mathbf{N}$ & $0.23637 \pm 0.00042$ & $0.41087 \pm 0.00061$ & 0.000000 & 0.27300 & $1.21050 \pm 0.03651$ \\
$\mathbf{O}$ & $0.23637 \pm 0.00042$ & $0.41087 \pm 0.00061$ & 0.000000 & 0.72700 & $1.21050 \pm 0.03651$ \\
\hline
\end{tabular}

Table S4. Atomic positions, occupancy, and thermal parameters for $x=4$.

\begin{tabular}{cccccc}
\hline $\mathbf{x}=\mathbf{4}$ & $\boldsymbol{x}$ & $\boldsymbol{y}$ & $\boldsymbol{z}$ & Occ. & Beq $\left(\boldsymbol{A}^{2}\right)$ \\
\hline Sr & 0.500000 & 0.500000 & 0.500000 & 1 & $2.36749 \pm 0.03445$ \\
$\mathbf{A l}$ & $0.19283 \pm 0.00065$ & $0.64267 \pm 0.00065$ & 0.000000 & 0.36592 & 1 \\
Li & $0.19283 \pm 0.00065$ & $0.64267 \pm 0.00065$ & 0.000000 & 0.63408 & 1 \\
$\mathbf{N}$ & $0.23794 \pm 0.00031$ & $0.40966 \pm 0.00045$ & 0.000000 & 0.23184 & $1.15107 \pm 0.02779$ \\
$\mathbf{0}$ & $0.23794 \pm 0.00031$ & $0.40966 \pm 0.00045$ & 0.000000 & 0.76816 & $1.15107 \pm 0.02779$ \\
\hline
\end{tabular}


Table S5. Atomic positions, occupancy, and thermal parameters for $x=5$.

\begin{tabular}{cccccc}
\hline $\mathbf{x}=\mathbf{5}$ & $\boldsymbol{x}$ & $\boldsymbol{y}$ & $\boldsymbol{z}$ & Occ. & Beq $\left(\boldsymbol{A}^{2}\right)$ \\
\hline Sr & 0.500000 & 0.500000 & 0.500000 & 1 & $2.18861 \pm 0.03780$ \\
Al & $0.19182 \pm 0.00080$ & $0.64604 \pm 0.00081$ & 0.000000 & 0.32489 & 1 \\
Li & $0.19182 \pm 0.00080$ & $0.64604 \pm 0.00081$ & 0.000000 & 0.67511 & 1 \\
$\mathbf{N}$ & $0.23741 \pm 0.00041$ & $0.40920 \pm 0.00056$ & 0.000000 & 0.14978 & $0.97043 \pm 0.03788$ \\
$\mathbf{O}$ & $0.23741 \pm 0.00041$ & $0.40920 \pm 0.00056$ & 0.000000 & 0.85022 & $0.97043 \pm 0.03788$ \\
\hline
\end{tabular}

Table S6. Atomic positions, occupancy, and thermal parameters for $x=6$.

\begin{tabular}{cccccc}
\hline $\mathbf{x = 6}$ & $\boldsymbol{x}$ & $\boldsymbol{y}$ & $\boldsymbol{z}$ & Occ. & Beq $\left(\boldsymbol{A}^{2}\right)$ \\
\hline $\mathbf{S r}$ & 0.500000 & 0.500000 & 0.500000 & 1 & $2.03456 \pm 0.06807$ \\
$\mathbf{A l}$ & $0.19136 \pm 0.00104$ & $0.64999 \pm 0.00107$ & 0.000000 & 0.29930 & 1 \\
Li & $0.19136 \pm 0.00104$ & $0.64999 \pm 0.00107$ & 0.000000 & 0.70070 & 1 \\
$\mathbf{N}$ & $0.23669 \pm 0.00051$ & $0.40879 \pm 0.00069$ & 0.000000 & 0.09860 & $0.67857 \pm 0.04508$ \\
$\mathbf{O}$ & $0.23669 \pm 0.00051$ & $0.40879 \pm 0.00069$ & 0.000000 & 0.90140 & $0.67857 \pm 0.04508$ \\
\hline
\end{tabular}

Table S7. O/N ratio and corresponding $z$ value in $\mathrm{Sr}_{0.98} \mathrm{Li}_{2.5+\mathrm{z}} \mathrm{Al}_{1.5-\mathrm{z}} \mathrm{O}_{3+2 \mathrm{z}} \mathrm{N}_{1-2 \mathrm{z}}: 0.02 \mathrm{Eu}$ for $x=3-6$.

\begin{tabular}{cccc}
\hline & Oxygen (\%) & Nitrogen (\%) & $\boldsymbol{z}$ \\
\hline $\mathrm{x}=3$ & 72.700 & 27.300 & -0.046 \\
$\mathrm{x}=4$ & 76.816 & 23.184 & 0.036 \\
$\mathrm{x}=5$ & 85.022 & 14.978 & 0.200 \\
$\mathrm{x}=6$ & 90.140 & 9.860 & 0.303 \\
\hline
\end{tabular}

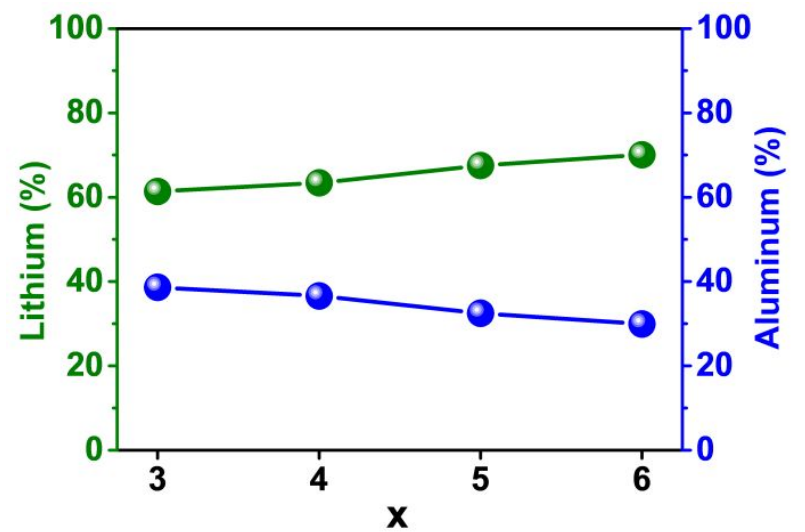

Figure S4. Occupancy value of $\mathrm{Li}$ and $\mathrm{Al}$ ions for $x=3-6$. 

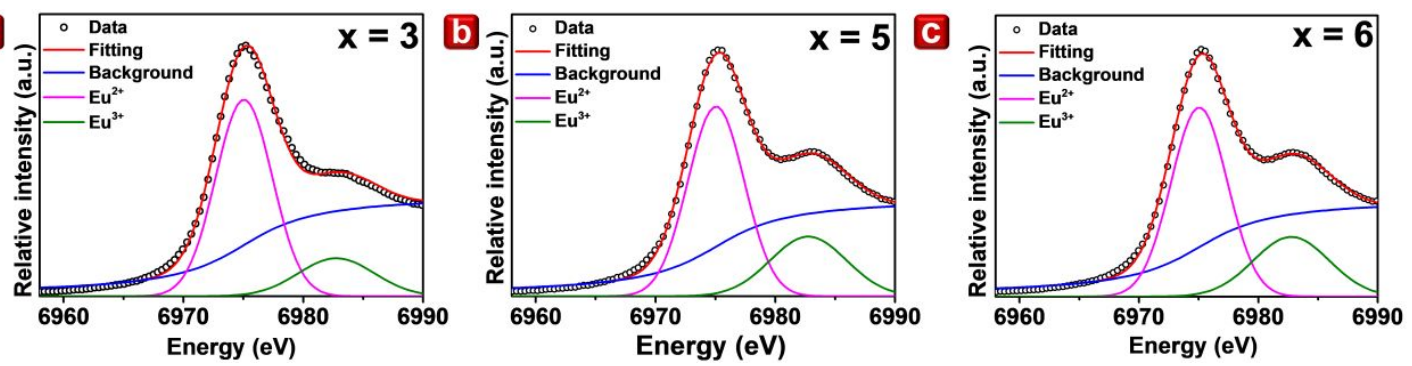

Figure S5. Fitting curves of $\mathrm{Eu}^{2+}$ and $\mathrm{Eu}^{3+}$ for $\mathrm{Eu} L_{3}$-edge XANES spectra of (a) $x=3$, (b) $x=5$, and (c) $x=6$.

Table S8. Ratio between $\mathrm{Eu}^{2+} / \mathrm{Eu}^{3+}$ for $x=3-6$.

\begin{tabular}{ccc}
\hline & $\mathrm{Eu}^{2+}$ & $\mathrm{Eu}^{3+}$ \\
\hline $\mathrm{x}=3$ & $79.4 \%$ & $20.6 \%$ \\
$\mathrm{x}=4$ & $75.1 \%$ & $24.9 \%$ \\
$\mathrm{x}=5$ & $70.3 \%$ & $29.7 \%$ \\
$\mathrm{x}=6$ & $70.2 \%$ & $29.8 \%$ \\
\hline
\end{tabular}
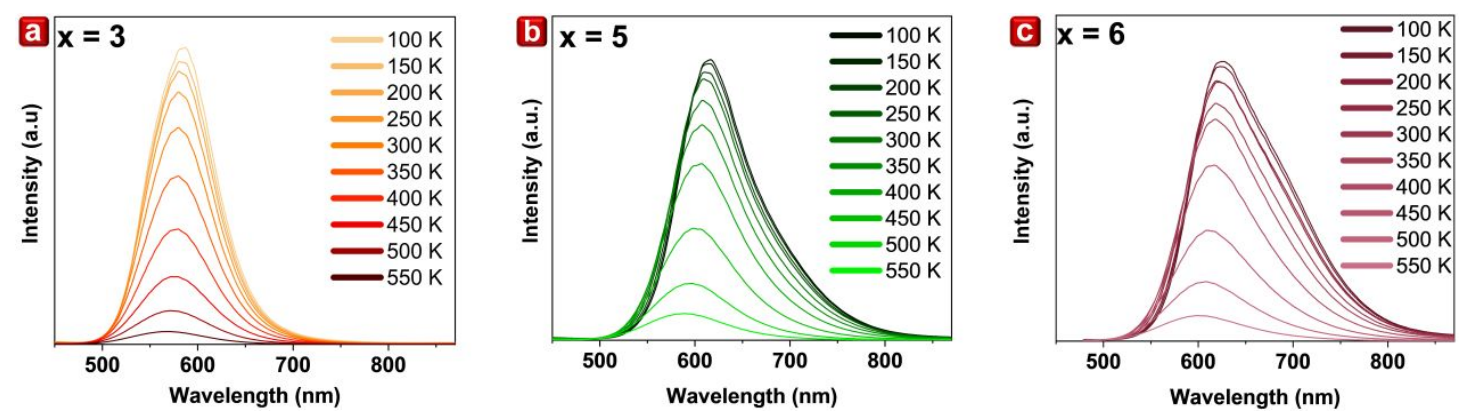

Figure S6. Temperature-dependent photoluminescence spectra of (a) $x=3$, (b) $x=5$, and (c) $x=6$.

Table S9. Parameters obtained from the fitting of temperature-dependent integrated intensity by using Equation (1). Parameters received from the fitting of temperaturedependent average decay time by using Equation (3) for $x=3-6$.

\begin{tabular}{ccccc}
\hline & $\begin{array}{c}\Delta E\left(\mathbf{c m}^{-1}\right) \\
\text { intensity }\end{array}$ & $\begin{array}{c}\mathbf{E}_{\mathrm{A}}\left(\mathbf{c m}^{-1}\right) \\
\text { decay time }\end{array}$ & $\mathbf{T}_{\mathbf{0}}(\boldsymbol{\mu s})$ & $\mathbf{p}_{\mathrm{nr}}\left(\mathbf{s}^{-1}\right)$ \\
\hline $\mathbf{x = 3}$ & $1690 \pm 130$ & $2379 \pm 200$ & $0.819 \pm 0.004$ & $8 \cdot 10^{8}$ \\
$\mathbf{x}=\mathbf{4}$ & $2380 \pm 130$ & $2959 \pm 330$ & $0.904 \pm 0.003$ & $3 \cdot 10^{9}$ \\
$\mathbf{x = 5}$ & $2590 \pm 170$ & $3268 \pm 220$ & $0.915 \pm 0.002$ & $6 \cdot 10^{9}$ \\
$\mathbf{x = 6}$ & $2770 \pm 200$ & $3351 \pm 150$ & $0.912 \pm 0.001$ & $7 \cdot 10^{9}$ \\
\hline
\end{tabular}



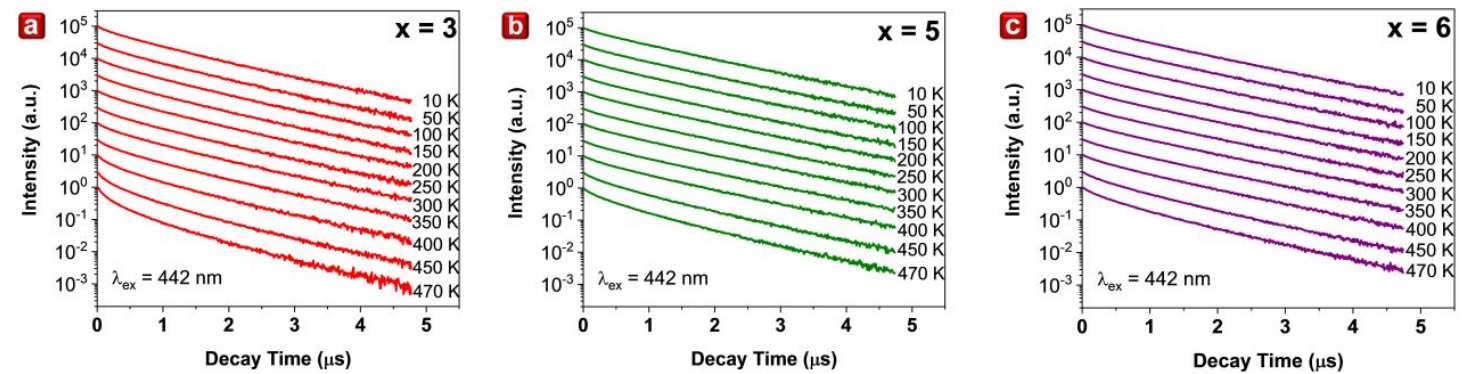

Figure S7. Temperature-dependent decay curves of (a) $x=3$, (b) $x=5$, and (c) $x=6$.
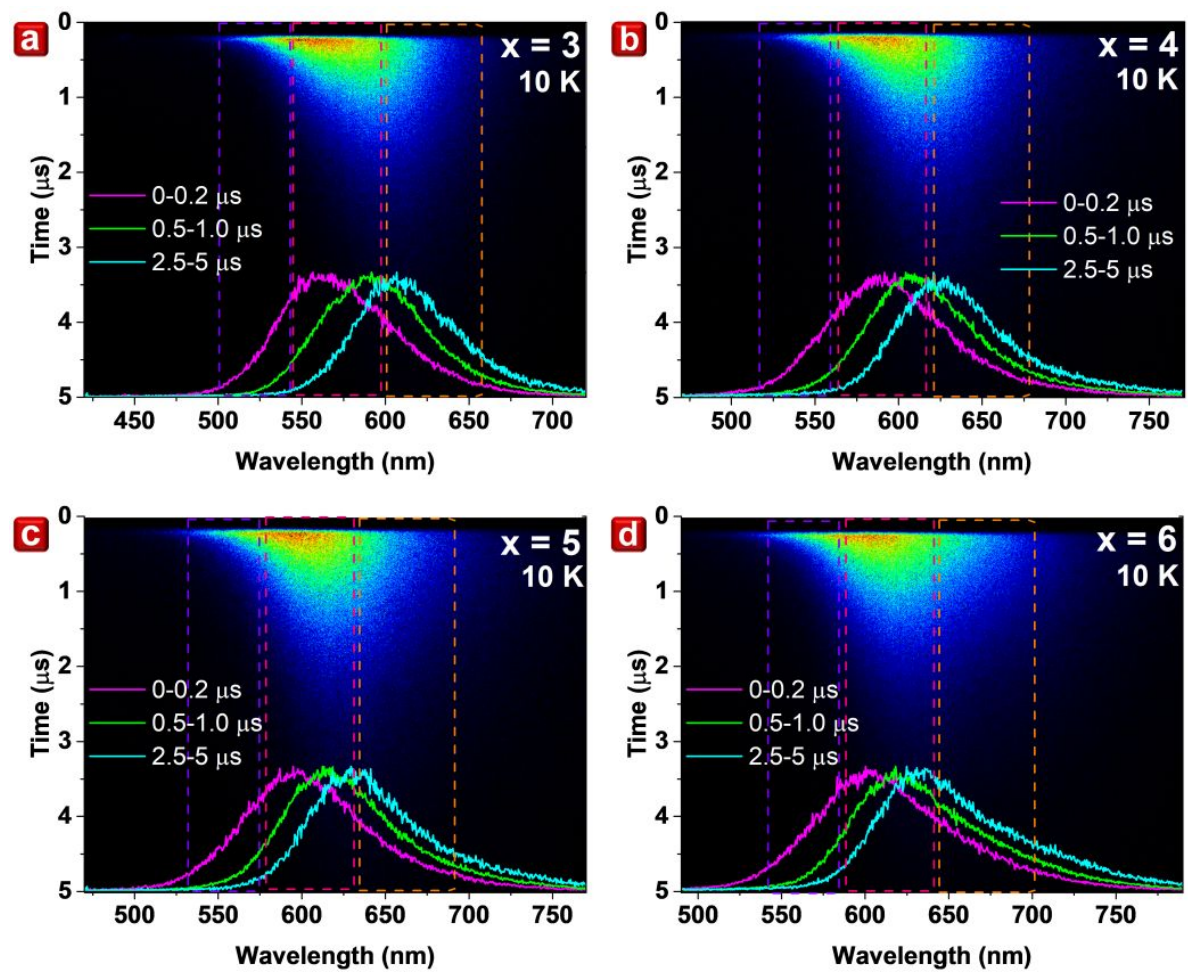

Figure S8. Time-resolved photoluminescence spectra of $0-0.2,0.5-1.0$, and 2.5-5 $\mu$ s ranges after laser pulsed and Streak camera image at $10 \mathrm{~K}$ for (a) $x=3$, (b) $x=4$, (c) $x$ $=5$, and (d) $x=6$. 

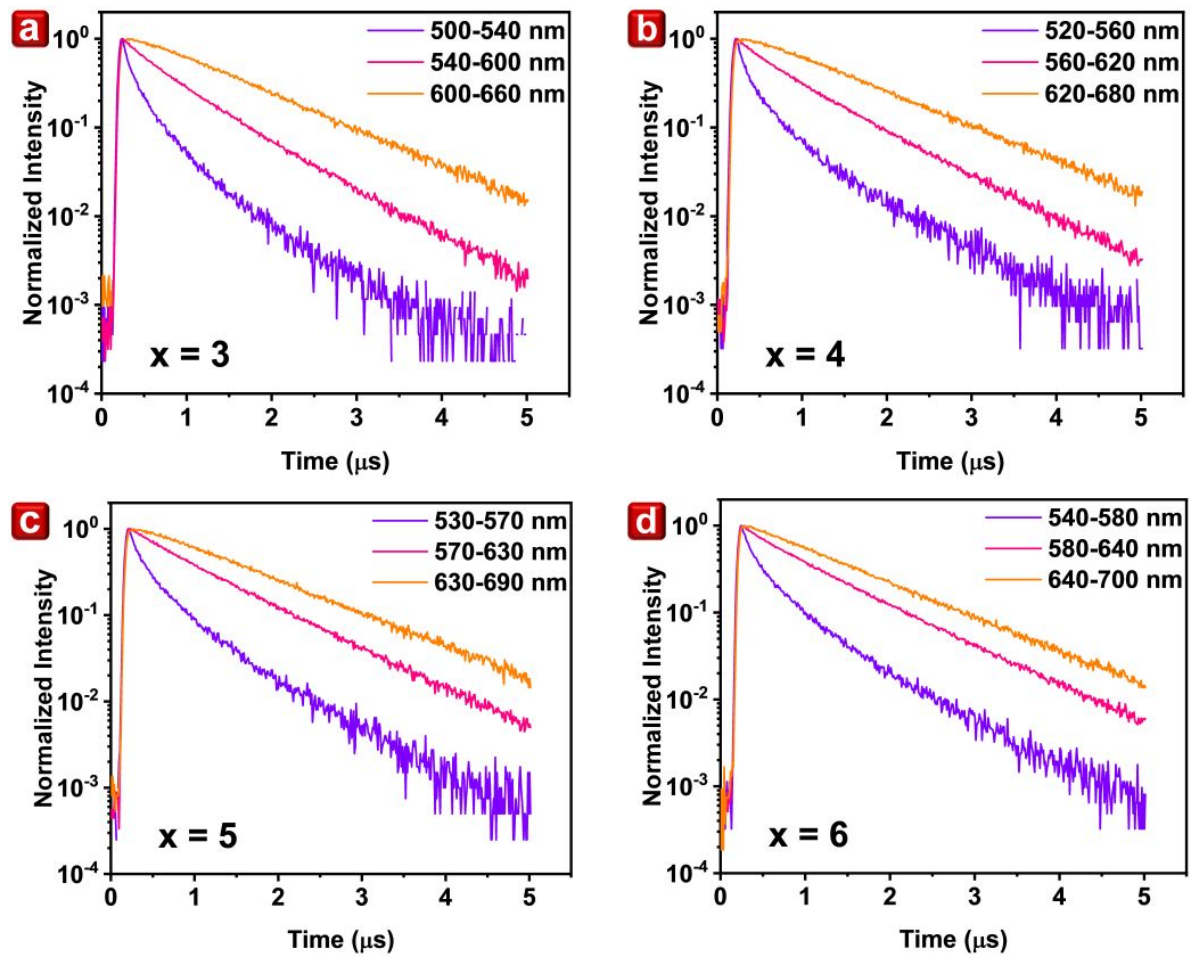

Figure S9. Decay profiles taken from the spectra range denoted by dashed contour in Figure S8 for (a) $x=3$, (b) $x=4$, (c) $x=5$, and (d) $x=6$. 